\title{
EL INTERCAMBIO Y COMPRA-VENTA DE TRABAJOS ACADÉMICOS A TRAVÉS DE INTERNET
}

\author{
Rubén Comas Forgas y Jaume Sureda Negre \\ rubencomas@uib.es; jaume.sureda@uib.es \\ Universidad de las Islas Baleares
}

\section{RESUMEN.}

En este artículo se presentan los resultados parciales de un estudio desarrollado por el equipo de investigación "Educación y Ciudadanía" de la Universidad de las Islas Baleares. El trabajo en cuestión forma parte del proyecto de I+D "El ciberplagio académico entre los estudiantes universitarios", financiado por el Ministerio de Educación y Cultura.

El contenido y la estructura formal de este documento se divide en tres apartados: 1) Intercambio y compra-venta de trabajos: claves para su análisis; 2) Estado de la cuestión, metodología y resultados de la investigación desarrollada; 3) Conclusiones, y discusión.

PALABRAS CLAVE: Integridad académica, Portales de compra-venta de trabajos, Fraude académico.

\section{ABSTRACT.}

This article presents the partial results of a study carried out by the research group "education and citizenship" based at the Balearic Islands University. The work in question is part of R+D project "The academic cyber-plagiarism among university students", funded by the Ministry of Education and Culture.

The content and formal structure of this document is divided into three sections: 1) Exchange and sale of works: keys to its analysis, 2) State of the art, methodology and research findings; 3) Conclusions, and discussion.

KEY WORD: Academic integrity, Paper mills, Academic fraud. 


\section{1) Intercambio y compra-venta de trabajos académicos: claves para su análisis}

El intercambio y la compra-venta de trabajos académicos prescritos por los profesores para aprobar una asignatura o curso no es una práctica nueva. Ahora bien, esta conducta, que atenta contra los principios de la integridad académica, ha aumentado enormemente a lo largo de los últimos años al amparo del desarrollo y penetración de las tecnologías de la información y comunicación (TIC). Sobre todo gracias al World Wide Web, lo que antes era un fraude de características artesanales ha ido adquiriendo dimensiones cuasi-industriales.

Diferenciamos, en nuestra propuesta, tres etapas en la evolución de las prácticas de comprar, vender e intercambiar trabajos académicos: una artesanal anterior a la aparición de Internet; una segunda fase propiciada precisamente por la aparición de la Red y dominada por los portales de intercambio de trabajos ya realizados $y$, finalmente, un tercer momento en el que, a través de Internet, se compran y venden trabajos académicos bajo demanda, hechos a medida según las necesidades específicas de cada usuario-consumidor.

\section{La etapa artesanal de la compra y venta de trabajos académicos}

La etapa que definimos como artesanal la situamos el periodo anterior a la aparición y desarrollo de Internet. Durante esta fase, quien quería adquirir un trabajo demandado por un profesor tenía que usar sus contactos personales para localizar a alguien que estuviera dispuesto a realizarlo a cambio de percibir una determinada compensación económica. Tenemos constancia documentada de estas prácticas en los años setenta del siglo pasado. Nos lo cuenta C.P., en la actualidad un reputado profesor universitario: "A principios de los años setenta yo estudiaba en la Universidad de Barcelona. Ya estaba en cuarto de carrera y vivía en un pequeño piso con otros estudiantes. Nuestra economía no era precisamente boyante y decidimos ofrecer nuestros servicios para realizar comentarios de texto a alumnos de cursos anteriores. En aquellos años, los comentarios eran unos ejercicios solicitados por muchos profesores. Yo me especialicé en comentarios de textos literarios, mientras que otro compañero hacía los trabajos de Filosofía. Dominábamos a la perfección la mecánica de los comentarios. Casi siempre conocíamos la obra sobre la que se nos pedía realizar un trabajo, pero si era necesario leíamos el libro que teníamos que comentar. En este último caso nuestras tarifas eran un poco más caras. En la memoria me ha quedado fijada la lectura rápida que tuve que hacer de una obra de Galdós; La de Bringas, es el título... Lo nuestro era como una pequeña mafia"

Por lo que comenta Umberto Eco (2001), este tipo de prácticas también se daban en ambientes académicos de la Italia del último tercio de siglo XX. Tal es así, que el propio Eco recomienda a aquellos estudiantes agobiados y desesperados por la necesidad de hacer una tesis que lo mejor 
que pueden hacer consiste en invertir "una suma razonable para encargar la tesis a otra persona", o que copien un trabajo "hecho unos años antes en otra universidad"1.

La aparición y universalización del World Wide Web, que dio comienzo en los años noventa del siglo pasado, cambió la forma de intermediación entre los autores (que ganaban en anonimato y aumentaban la potencial demanda de sus servicios) los vendedores (que ganaban en comodidad a la hora de sacar la oferta al mercado y aumentaban su potencial número de clientes) y los compradores (que veían aumentada exponencialmente la oferta, la rapidez en el acceso a ésta y la comodidad para hacerse con trabajos elaborados por otros ${ }^{2}$ ).

\section{La etapa de los bancos o fábricas de ensayos}

En la segunda mitad de los años 90 aparecieron los llamados "bancos o fábricas de ensayos" ${ }^{3}$; portales que facilitan el intercambio -ya sea de forma gratuita o mediante pago- de trabajos ya elaborados -en ocasiones ya utilizados- y que, en el mejor de los casos, el alumnado, haciendo uso de los comandos "copiar" y "pegar", reelabora de acuerdo con sus intereses o necesidades. En el peor de los casos, simplemente, y siguiendo el modelo de la etapa artesanal, se entregan los trabajos localizados sin cambio significativo alguno como si la autoría fuese suya. En la actualidad sigue existiendo una gran cantidad de este tipo de portales, sumamente populares entre los estudiantes $^{4}$

La proliferación del uso por parte de los estudiantes de portales que ofrecen el intercambio de trabajos académicos ya realizados se ha visto parcialmente restringido a causa de los mecanismos de control anti-plagio que muchos centros educativos, especialmente del entorno cultural anglosajón, han puesto en marcha desde finales de los años 90. Efectivamente, en estas fechas se llegó a una situación tan escandalosamente preocupante por el uso por parte del alumnado de la información accesible a través de Internet $y$, sobre todo, por su habilidad en el uso de los comandos "copiar" y "pegar" a la hora de realizar sus trabajos académicos que se tuvieron que buscar remedios. Fue así como aparecieron programas y servicios de detección del plagio. Uno de los más populares, Turnitin -software con patente norteamericana y usado por numerosos centros académicos de los EEUU y el Reino Unido-, afirma comprobar cada día más de 40.000

1 Evelyn Waugh señala en su autobiografía que en la escuela Lancing, un centro perteneciente a una fundación de colegios concebidos para inculcar los preceptos de la Iglesia Anglicana y en el que estuvo internado cuando tenía trece años -hablamos pues de 1916- era corriente "que los chicos pequeños, los más listos, se ganasen los favores de los más grandes, y más estúpidos, haciéndoles los ejercicios" (Waugh, 2007)

2 A mediados del siglo XIX, en EEUU, ya se comercializaban trabajos académicos: "Students who were so inclined in the mid-1800s could easily obtain completed papers from fraternity houses or "term-paper mills” that set up shop near many universities" (Hansen, 2003)

3 Los conceptos más utilizados en inglés para referirse a estos bancos de ensayos son: Paper mills, Term paper mill databases, Pre-written term papers. Library of essays. No existe unanimidad sobre cuál fue el primer banco o fábrica de ensayos. Pero sin duda Schoolsucks, puesto en marcha en junio de 1996, fue uno de los pioneros y su rápida popularidad contribuyó decisivamente a la expansión de muchos otros (Leland, 2002). Un interesante trabajo sobre este tema es el titulado Cheating Goes Hi-Tech: Online Term Paper Mills (Campbell, Swift, \& Denton, 2000).

4 En el trabajo Cheating 101:Paper Mills and You (Fain \& Bates, 2003) se ofrece un listado de más de 60 portales de estas características, especializados en diversas áreas y temas. En ambientes hispanohablantes son muy conocidos los servicios que se ofrecen desde portales como El rincón del vago (http://www.elrincondelvago.com) -imposible una descripción más explícita y exacta de sus usuarios-, Alipso (http://www.alipso.com) o Monografías (http://www.monografias.com). 
trabajos académicos, de los cuales un 30\% -según datos de la propia empresa- suele presentar indicios de plagio (Maxymuk, 2006)

\section{La etapa de las fábricas de ensayos a la carta}

Las técnicas defraudadoras, como los malos virus, se adaptan a las nuevas situaciones y estímulos. Cuando los autores y vendedores encubiertos se plantearon cómo superar la barrera impuesta por los programas detectores de plagio, encontraron una respuesta sencilla: ofrecer trabajos originales capaces de superar la prueba de cualquier programa anti-plagio. Es decir: si en la primera etapa, la artesanal, la suplantación era la táctica usada por los defraudadores, y en la segunda lo fue el plagio -ya sea copiando de aquí y de allá o reproduciendo trabajos enteros-, en esta tercera fase se vuelve a recurrir a la suplantación, a la realización de trabajos a la carta; trabajos originales hechos según la demanda y que pueden superar la prueba de cualquier programa informático detector de la copia ${ }^{6}$. En esta nueva situación, sin embargo, la suplantación adopta un nuevo enfoque: "tecnifica" y en cierta forma formaliza la relación entre la oferta y la demanda mediante la intermediación de portales en Internet. Los pequeños bares de las facultades, que en la etapa que hemos definido como artesanal eran los escenarios en los que los estudiantes buscaban a quién, por una determinada cantidad de dinero, estuviera dispuesto a hacer el trabajo solicitado por un profesor, se han visto sustituidos por otros espacios sin fronteras ni límites aparentes: los escenarios que posibilita Internet. En la Red han aparecido una gran cantidad de portales -verdaderas fábricas de trabajos académicos- que ofrecen la realización de trabajos de todo tipo. Las reglas son claras: tarifas con precios diferentes dependiendo del encargo -no se cobra lo mismo por realizar una tesis doctoral que por hacer un trabajo de curso de bachillerato- y garantías de que se entregará un producto de calidad. Evidentemente, todo se rige por las reglas del mercado: el cliente puede elegir diferentes niveles de calidad -no puede valer lo mismo un aprobado que un sobresaliente- y la premura tiene también su precio: no se cobrará lo mismo por un trabajo si se tiene que entregar dentro de dos meses o si se necesita para ser presentado en dos días.

En todo caso, y en forma de secuencia circular, el sistema de fraude académico pasó de la suplantación de los años setenta al plagio e intercambio de los noventa y, poco a poco, parece estar pasando nuevamente del plagio a la suplantación ${ }^{7}$. Las transición de la primera a la segunda etapa ha sido posible, en gran medida, gracias al desarrollo, implantación y universalización de las

5 Otra consecuencia de esta toma de conciencia del problema del plagio fue la asunción por parte de los servicios bibliotecarios de muchas universidades de la responsabilidad de difundir principios éticos en el uso de la información. Así fue como en las Web de las bibliotecas empezaron a dedicar páginas para formar e informar sobre el plagio académico tanto al alumnado como al profesorado con el objetivo de no incurrir en él.

6 Existe también una modalidad reciente que se basa en la traducción de trabajos académicos de un idioma (en el que originalmente se realizó el trabajo) a otro que será en el que se venderá y entregará como original y nuevo.

7 El paso de una etapa a otra no implica, ni mucho menos, la desaparición de las prácticas de suplantación o plagio de la anterior; sino que supone la acumulación de todas ellas. Es decir: la aparición de las fábricas de ensayos a la carta no ha provocado la desaparición del uso de los bancos de ensayos ya elaborados ni siquiera las prácticas de la compra-venta de trabajos en la modalidad que hemos definido de artesanal. Si que es cierto, sin embargo, que todas las formas de plagiar se han visto favorecidas por Internet. Un ejemplo de la pervivencia y profundidad de esta modalidad la tenemos en el programa que la cadena ABC emitió en el 2004 titulado Caught Cheating (Paul, G. 2004) En este programa se muestra a un estudiante que afirmaba haber realizado más de 500 trabajos académicos para otros alumnos, llegando a cobrar unos 25 dólares por página redactada. No comercializaba sus trabajos a través de un portal de Internet sino con el boca a boca característico de la primera etapa. Pero sí que se aprovecha de Internet para documentarse, buscar trabajos ya hechos y maquillarlos como si fueran nuevos. 
$\mathrm{TIC}^{8}$; este hecho es evidente a la hora de encontrar explicaciones al paso de la etapa "artesanal" basada en la búsqueda de "ghost writers" anónimos en entornos próximos y conocidos- a la de los portales de trabajos "precocinados" que en la mayoría de los casos eran usados varias veces por más de un alumno -los "bancos de ensayos"-, que provocaron la eclosión de la práctica basada en el plagio mediante el uso de los comandos de los editores de texto "copiar" y "pegar". Distinto ha sido el origen y causas de la transición de la etapa del plagio e intercambio de trabajos, a la de la nueva suplantación basada en portales de Internet y la compra-venta de trabajos realizados a medida; en este caso la causa principal ha sido la necesidad de eludir los sistemas de control antiplagio instaurados en muchos centros educativos. La utilización de programas informáticos puestos en marcha para detectar el plagio, así como la instauración de otros sistemas de control, han provocado que la suplantación dura y pura volviese a adquirir carta de naturaleza en las aulas. Suplantación, que, enmarcada en coordenadas especificas propias de la sociedad de la información, adquiere en estos momentos descomunales dimensiones. Enfrentarse a la nueva suplantación ${ }^{9}$ supondrá poner en marcha nuevas estrategias basadas en conocer el qué, el por qué y el cómo de la situación originada.

\section{Principales características de los portales de intercambio y compra-venta de trabajos académicos}

Los nuevos portales que ofrecen trabajos académicos a la carta se rigen por las reglas del mercado. Su gran proliferación y los datos económicos que se conocen -sólo en el Reino Unido estas empresas mueven más de 200 millones de libras esterlinas al año ${ }^{10}$ - indican que se trata de un negocio boyante. Y como todo buen negocio que compite para atraer clientes se preocupa por publicitar sus servicios de la mejor manera posible. Con el fin de convencer de sus excelencias, las empresas que dirigen estos portales no sólo contratan la inclusión de anuncios en Google ${ }^{11}$, sino que en la página principal colocan diversos y curiosos eslóganes de marketing a modo de anzuelo.

\footnotetext{
8 La venta de títulos académicos a través de las llamadas “fábricas de títulos” (Diploma mills o Degree mills) constituye otro de los fraudes académicos que se han visto especialmente propiciados por el desarrollo de las TIC. Se trata de personas u organizaciones no acreditadas que venden diplomas académicos según demanda del cliente. Los interesados pueden obtener el título que piensen que se merecen o que necesitan para progresar en su puesto de trabajo; el único requerimiento consiste en pagar la tarifa estipulada que puede oscilar entre los 300 y los 12.000 dólares. En relación al tema véase el portal del Centre for Information on Diploma Mills con extensa información sobre este fraude: http://www.diplomamills.nl/index engels.htm Una página con una excelente información sobre las fábricas de títulos es también la de la Student Assistance Commission, del Estado de Oregón: http://www.osac.state.or.us/oda/diploma_mill.html
}

9 En la literatura existente sobre el tema, la práctica de comprar trabajos académicos ya realizados se suele incluir en la categoría de plagio o ciberplagio. pero nosotros coincidimos con Page (2004) cuando éste propone separar esta práctica del ciberplagio y usar el nombre de "Cyberpseudepigraphy9” (Ciber suplantación) para diferenciarla del plagio. En realidad no se copia: se suplanta la autoría.

10 Se conocen pocos datos sobre el movimiento e impacto económico de estas empresas. Dorit Chomer, director de una de estas compañías, afirmó, en una entrevista radiofónica realizada en el 2005, que su empresa vendía entre 500 y 1000 ensayos semanales "sobre todo a estudiantes extranjeros en el Reino Unido”, los cuales representan el 80\% de sus clientes. (Levinson, 2005) En un reciente artículo publicado en la Web de Higher Education \& Research Opportunities in the United Kingdom, HERO, se señala que, según fuentes gubernamentales del Reino Unido, el negocio de la venta de ensayos online anualmente mueve unos 200 millones de libras. Según este mismo trabajo, una de las compañías más prolíficas ha afirmado mover al año 1'6 millones de libras (HERO, 2007).

11 En el mes de Junio de 2007 la dirección de Google decidió dejar de incluir en sus páginas anuncios de compañías que venden ensayos y tesis; una medida recibida con satisfacción por las autoridades académicas del Reino Unido. Hasta esta fecha, este tipo de empresas se anunciaban con toda libertad. 
Veamos algunos de ellos: "You tell the story... I paint your perfect picture "12" "Let professionals take care of your papers ${ }^{13 "}$ "A good thesis is a done thesis ${ }^{14 "}$ "The difference between passing and failing a course" Las explicaciones que en algunos casos acompañan a los eslóganes son más explícitas y reveladoras de la verdadera naturaleza de los servicios que ofrecen: Having difficulty in writing your disseration?... Worry no more (...) You only need to send us your desired topic and just sit back and relax, while we provide you with the best dissertation! $!^{15}$ We are here to assist individuals who are having a hard time finishing their assignment. We can write on all topics and subjects. All we need is for you to provide us your instructions and we write a paper from it. ${ }^{16}$ We write the assignment for you ${ }^{17}$ The "writing team" consists of 1 research specialist, and 1 professional writer. They will do the job for you $!^{18}$

Realizar trabajos académicos para que los clientes-alumnos los presenten como si fueran suyos es el principal servicio que ofrecen estos portales. Aunque también es cierto que algunos de ellos una ínfima minoría- dicen limitarse a la simple asesoría: "Our firm can help you with any thesis or dissertation. Of course, we cannot do all the work for you and conduct all studies because a dissertation, typically, is a culmination of years of academic study. Nevertheless, we have writers with profound expertise in your specific area of study, and their input will be invaluable to your dissertation"19 "We provide you with the guidance, feedback, and partnership you need to graduate on time and get on with your life and career $^{\prime 20}$. La frontera entre lo que podríamos considerar, por una parte, oferta de suplantación y, por otra, servicios de asesoramiento es nítida cuando se aborda desde una perspectiva teórica. En la práctica, sin embargo, la distinción es más difusa. En todo caso hay que constatar que algunos de los portales localizados y analizados se presentan no tanto como servicios de suplantación como de asesoramiento ${ }^{21}$. Otros portales, quizás para intentar limpiar su mala conciencia e imagen, ponen en su Web observaciones como ésta: You can use it -se refiere a los trabajos ofrecidos por el portal- for personal research purposes, or as a model answer for your own paper. What you cannot use it for, however, is to submit it to your university as your own paper. This is a common policy for all the reputable essay companies in the UK (...) We are a company that offers help to students who cannot complete their projects on their own ${ }^{22}$. Otros portales ofrecen la suplantación como segunda alternativa: En Redacción de Tesis ${ }^{23}$ podemos leer: "Ofrecemos asesoramiento para cualquier tipo de trabajo académico: anteproyectos, proyectos, tesis, tesinas, monografías, trabajos prácticos, etc. O bien, realizamos total o parcialmente alguno de los tipos de trabajo mencionados". En resumidas cuentas, los servicios que se ofrecen a través de estos portales son dos. El primero es el de la

\footnotetext{
12 From Your Editor Jill S. Eastwood. http://www.jeastwood.com/

13 http://www.1stessays.com/

14 http://www.tadafinallyfinished.com/index0307C.html

15 Dissertationassistant

16 Cebuecommerce

17 Essays on the dot

18 Dissertations \& Assignments

19 http://www.customessaymeister.com

20 http://www.dissertationadvisors.com/index.shtml

21 Es el caso, por ejemplo, de Dissertation advisors. Este servicio facilita un listado de “consejeros” a quienes dirigirse si un estudiante tiene dudas a

la hora de realizar un trabajo

22 http://www.dissertationsandassignments.com/

23 http://ar.geocities.com/ayudatesis/index.html
} 
suplantación dura y pura para la presentación de trabajos académicos. Esta suplantación se ofrece mediante la realización de trabajos a la carta, aunque algunos de estos portales también los comercializan "pre-cocinados", es decir; trabajos ya listos que pueden adaptarse a las necesidades del estudiante comprador, pero que no se hacen a medida, siguiendo el modelo dominante en la segunda etapa de la evolución de la compra-venta de trabajos académicos a la que nos hemos referido en el anterior apartado. El segundo servicio que se ofrece se limita al asesoramiento para la realización de trabajos académicos -evaluación de viabilidad de proyectos, asesoramiento metodológico, etc.- o a su corrección.

Los trabajos que se ofrecen abarcan todas las áreas y se dirigen básicamente a estudiantes de secundaria y universidad. La tipología de productos ofertados responde a todas las posibles demandas que un profesor puede prescribir a sus alumnos: ensayo, recensión de un libro, propuesta de investigación, tesis, estudio de un caso, etc. La oferta se adapta a la demanda. $Y$ como no podía ser menos, algunos portales se han especializado en determinados clientes, sobre todo los extranjeros a los que se les dice que se tendrá en cuenta el "toque" cultural propio de su entorno a la hora de redactar su trabajo.

El precio depende del tipo de trabajo solicitado y del tiempo que se dispone para realizarlo. No se aplica la misma tarifa para un ensayo que urge para pasado mañana que si se necesita para dentro de un mes. Por otra parte, muchos portales permiten que el cliente elija la calidad del trabajo solicitado -normal, media y superior, suelen ser las categorías ofrecidas-. Quizás pensarán, guiados por las leyes del mercado, que no tiene que costar lo mismo un notable que un aprobado. Combinando estos tres factores -calidad, calendario y tipología- se establece la tarifa. Para un trabajo de curso a entregar en 7 días y de alta calidad se cobra unos 19 dólares por página ${ }^{24}$; por una memoria de investigación a entregar dentro de 30 días la tarifa puede oscilar entre los 18 dólares por página ${ }^{25}$ y los $12^{\prime} 95^{26}$. Las urgencias se pagan caras: si se necesita un trabajo antes de 24 horas la página le costará al cliente 24 dólares. No todos los portales tienen el mismo sistema tarifario: algunos envían el presupuesto una vez recibido el encargo, mientras que otros -es el caso de Cebue Comerce ${ }^{27}$ - incluso tienen una calculadora de tarifas en su página Web para que el cliente sepa de forma inmediata por cuanto le saldrá el trabajo que necesita.

En los últimos tiempos han empezado a surgir -fruto del fenómeno globalizante- gran cantidad de portales establecidos en países como India, China o Vietnam que ofrecen sus servicios al mercado anglosajón por unos precios muy bajos. Algunos de estos portales dan gato por liebre; trabajos copiados o reciclados, redactados de forma deficiente, etc. Esto ha provocado que los portales establecidos fuera de los EEUU hayan pasado a ser sospechosos, a ser considerados productos de baja calidad y fraudulentos -¿acaso no lo son todos?- a los que hay que dar caza. Con este fin son muchos los portales o empresas "clásicas" que incorporan indicaciones sobre cómo detectar a los 
impostores. Éstos enumeran una serie de recomendaciones para detectar los portales extranjeros. Hay, incluso, un Ilamado "observatorio" EssayFraud (http://www.essayfraud.org) que señala hasta 11 indicadores para detectar a los portales extranjeros - para ellos sinónimo de fraude- y que van desde la calidad del inglés utilizado en el portal hasta los prefijos de los números de fax o de teléfono, pasando por el precio: según este "observatorio", no se puede "comprar" un trabajo académico serio por menos de 16 dólares la página. Curioso observatorio para el cual el problema parece residir no tanto en la existencia de estos portales como en aquellos que no ofrecen un servicio de calidad. La ética académica parece que se limita a la impuesta por el mercado; lo importante consiste en crear una potente industria de construcción y alquiler de trabajos académicos.

Estos sitios también ofrecen información sobre algunas de las características de los autores de los trabajos: "100\% graduados en Universidades americanas y en ningún caso asiáticos o ciudadanos de la Europa del Este", se señala en una de las páginas de Meister Custom Essay ${ }^{28}$. "Our writers are professional writers. They write for a living. Majority of our writers hold a Bachelor's Degree in different disciplines, some of our writers posses a Master's Degree in diverse disciplines, and we have a couple of writers who hold Doctorate Degrees", se asegura en Alessays.com ${ }^{29}$. En Dissertation Writing ${ }^{30}$ se afirma que "The writers and professors at Dissertation-Writing-Online are specialists in dissertation writing. They hold PhD degrees and have years of credible writing and teaching experience ". Hay portales que incluso proporcionan información sobre el número de autores con los que trabajan: es el caso de Dissertations Experts que afirma contar con más de “... 200 university professors and instructors, professional editors proficiently trained in writing, researching, editing in a wide range of disciplines and subject areas" ${ }^{\prime 31}$. Llama la atención que algunos portales -es el caso de Dissertations and Assignments- ofrecen trabajo: "We are always looking for graduates who have a good degree to fill positions. We offer a number of vacancies for graduates who can work as freelance writers in all academic areas. You should have a first class degree to apply. If we take you onboard, you can work from home, but we will have high expectations of you "32 Aparte de lo que se dice en los portales -obviamente que cuentan con excelentes profesionales- se puede pensar que no son pocos los estudiantes que se dedican a estos menesteres.

La dinámica, el modus operandi, de estos portales es prácticamente el mismo en todos ellos: el cliente tiene que rellenar un formulario señalando el tipo de trabajo que desea, el tema, el número de páginas, el nivel educativo en el que lo va a presentar. En algunos casos puede, incluso, solicitar especificidades de su demanda como, por ejemplo, si el trabajo debe tener un limitado número de citas o, como ya hemos señalado, si debe incluir algún "retoque" cultural o lingüístico que no le haga levantar sospechas. Cuando el pedido llega a la empresa, se hace un presupuesto en algunos portales puede hacerse de forma automática como se ha comentado- el cual deberá

28 http://www.customessaymeister.com/ Consultado el 29 de octubre de 2007

29 http://www.a1essays.com/staff/default.html Consultado el 29 de octubre de 2007

30 http://www.dissertation-writing-online.com/

31 http://www.dissertationsexperts.com/aboutus.php Consultado el 29 de octubre de 2007

32 http://www.dissertationsandassignments.com/jobs.asp Consultado el 29 de octubre de 2007 
ser aceptado por el cliente. A continuación, se asigna un autor especialista en la materia el cual realizará el trabajo. Una vez finalizado, para evitar sorpresas, éste será evaluado por un programa antiplagio certificando así su originalidad.

\section{2) Estado de la cuestión, metodología y resultados de la investigación}

La pregunta que parece adecuado plantearse llegados a este momento es: ¿Cuál es la magnitud del fenómeno relatado? La respuesta, como casi siempre, no puede ser $100 \%$ categórica. Podemos, eso sí, hablar de tendencias. Los datos de que se dispone sobre la prevalencia de los diversos tipos de plagio y otras formas de deshonestidad académica por parte de los estudiantes universitarios señalan que la compra de trabajos a través de Internet se sitúa en tono a un $5 \%$. Según un trabajo de investigación llevado a cabo en la Universidad de Lyon, el 4’3 por ciento del alumnado afirmaba haber comprado a través de Internet trabajos académicos ya realizados (Université de Lyon, 2007) ${ }^{33}$. Los estudios desarrollados sobre esta temática en EEUU muestran una mayor prevalencia: entre un $16 \%$ según un macro-estudio del profesor MacCabe (McCabe, 2001) y un 16’3\% según la encuesta de Scalon y Neumann (Scanlon \& Neumann, 2002) aplicada en el curso 1999-2000 al alumnado de 9 instituciones de enseñanza superior norteamericanas. Otros trabajos realizados en EEUU, con grupos más reducidos, como es el caso de una encuesta con alumnos de ingeniería (Carpenter et al., 2006), ofrecen datos muy parecidos a los obtenidos en la Universidad de Lyón: el 4’5\% de los alumnos afirmó haber comprado trabajos ya hechos.

\section{Metodología del estudio:}

Los resultados que aquí se presentan se obtuvieron a partir de la administración de un cuestionario a 2 grupos de universitarios: Grupo 1 (Alumnado de la UIB); Grupo 2 (Alumnado de diversas universidades españolas registrados en el portal Universia)

Los datos fueron obtenidos mediante la aplicación de una encuesta ${ }^{34}$ cuyas principales características son:

Universo:

Grupo 1: Alumnado de primer y segundo ciclo de la Universidad de les Islas Baleares (11.797 alumnos) $)^{35}$

Grupo 2: Alumnado registrado en el portal UNIVERSIA (37.490 usuarios).

Afijación:

Grupo 1: Proporcional

34 El cuestionario utilizado fue testado con anterioridad a la implementación del trabajo de campo; el test consistió en pasar el cuestionario entre 32 alumnos de primer y segundo curso de la Diplomatura de Educación Social de la UIB.

35 Fuente: Vicerrectorado de alumnado de la UIB (curso 2005-2006) 
Grupo 2: Aleatoria

Muestra:

Grupo 1: 727 unidades de análisis (cuestionarios), resultando un error muestral para el conjunto de la muestra del 3,52\%, estimado para un nivel de confianza del $95 \%$ y bajo la condición más desfavorable de $\mathrm{p}=\mathrm{q}=0.05$.

Grupo 2: 560 unidades de análisis (cuestionarios), resultando un error muestral para el conjunto de la muestra del $4,11 \%$, estimado para un nivel de confianza del $95 \%$ y bajo la condición más desfavorable de $\mathrm{p}=\mathrm{q}=0.05$.

Fecha del trabajo de campo:

Grupo 1: Mayo-Junio de 2007

Grupo 2: Abril-Junio de 2007

Proceso de datos:

Los datos obtenidos fueron analizados con el paquete estadístico: SPSS 14.

\section{Resultados ${ }^{36}$ :}

Ambos grupos debían responder a una serie de cuestiones de manera dual. Esto es, por un lado manifestando su práctica personal respecto de diferentes acciones contra la integridad académica, y en segundo lugar opinando acerca de los comportamientos del resto de universitarios sobre los mismos planteamientos.

Presentar como propios trabajos completos obtenidos a través de Internet

Poco más de uno de cada diez alumnos de la UIB $(10,4 \%)$ y el $6 \%$ del alumnado registrado en UNIVERSIA (6,6\%) manifiesta haber descargado de la Red un trabajo académico completo y haberlo entregado sin cambios como si fuera propio (ver Tabla 1).

Tabla 1: Descarga de trabajos completos de Internet

\begin{tabular}{|l|lr|l|l|l|}
\hline Grupo & $\begin{array}{l}\text { Nunca } \\
\text { "descargado" han }\end{array}$ un & Han & "descargado" & Han & “descargado" \\
trabajo de Internet y lo & un trabajo de & un trabajo de & "descargado" \\
un trabajo de \\
han entregado sin & Internet y lo & Internet y lo & Internet y lo \\
cambios como si fuera & han & han & han \\
propio. & entregado & entregado & entregado \\
& sin cambios & sin cambios & sin cambios \\
como si fuera & como si fuera & como si fuera \\
\hline
\end{tabular}

\footnotetext{
${ }^{36}$ En este artículo únicamente se presentan los resultados obtenidos en la investigación que tienen que ver con compra-venta de trabajos y descarga de trabajos completos de Internet. El estudio en que se enmarcan estos los datos obtenidos es mucho más amplio y examina de manera global la Integridad Académica.
} 


\begin{tabular}{|l|l|l|l|l|}
\hline & & $\begin{array}{l}\text { propio entre } \\
\mathbf{1} \text { y } 2 \text { veces }\end{array}$ & $\begin{array}{l}\text { propio entre } \\
\mathbf{3} \text { y } \\
\text { ocasiones }\end{array}$ & $\begin{array}{l}\text { propio en 5 o } \\
\text { más de 5 } \\
\text { ocasiones }\end{array}$ \\
\hline $\begin{array}{l}\text { Grupo } \\
\text { Alumnado UIB }\end{array}$ & $89,6 \%$ & $7,6 \%$ & $1,8 \%$ & $1,0 \%$ \\
\hline $\begin{array}{l}\text { Grupo } \\
\text { Alumnado } \\
\text { registrado en } \\
\text { UNIVERSIA }\end{array}$ & $93,4 \%$ & $4,2 \%$ & $2,6 \%$ & $0,8 \%$ \\
\hline
\end{tabular}

EI $70 \%$ de estudiantes de la UIB y el $62,1 \%$ de UNIVERSIA consideran que, en al menos una ocasión, sus compañeros en la universidad han "descargado" y/o copiado íntegramente trabajos académicos de la Red y los han presentado como si fueran propios (ver Tabla 2).

Tabla 2: Descarga de trabajos completos de Internet (opinión de las prácticas del resto de alumnado)

\begin{tabular}{|c|c|c|c|c|}
\hline Grupo & $\begin{array}{l}\text { Creen que sus } \\
\text { compañeros } \\
\text { nunca han } \\
\text { "descargado" } \\
\text { un trabajo de } \\
\text { Internet y lo } \\
\text { han entregado } \\
\text { sin cambios } \\
\text { como si fuera } \\
\text { propio }\end{array}$ & $\begin{array}{l}\text { Creen que sus } \\
\text { compañeros } \\
\text { esporádicamente } \\
\text { han "descargado" } \\
\text { un trabajo de } \\
\text { Internet y lo han } \\
\text { entregado sin } \\
\text { cambios como si } \\
\text { fuera propio. }\end{array}$ & $\begin{array}{l}\text { Creen que sus } \\
\text { compañeros } \\
\text { frecuentemente } \\
\text { han } \\
\text { "descargado" un } \\
\text { trabajo de } \\
\text { Internet y lo han } \\
\text { entregado sin } \\
\text { cambios como si } \\
\text { fuera propio }\end{array}$ & $\begin{array}{l}\text { Creen que sus } \\
\text { compañeros muy } \\
\text { frecuentemente } \\
\text { han } \\
\text { "descargado" un } \\
\text { trabajo de } \\
\text { Internet y lo han } \\
\text { entregado sin } \\
\text { cambios como si } \\
\text { fuera propio }\end{array}$ \\
\hline $\begin{array}{ll}\text { Grupo } & 1 \\
\text { Alumnado UIB }\end{array}$ & $30,0 \%$ & $44,5 \%$ & $20,2 \%$ & $5,3 \%$ \\
\hline $\begin{array}{l}\text { Grupo } \\
\text { Alumnado } \\
\text { registrado en } \\
\text { UNIVERSIA }\end{array}$ & $37,9 \%$ & $33,7 \%$ & $18,1 \%$ & $10,3 \%$ \\
\hline
\end{tabular}


Compra de trabajos académicos a través de Internet

El 4,7\% del alumnado de la UIB y el 3,2\% de los universitarios registrados en UNIVERSIA dicen haber comprado, en al menos una ocasión, un trabajo académico a través de un portal de Internet (ver Tabla 3).

Tabla 3: Compra de trabajos académicos de Internet

\begin{tabular}{|c|c|c|c|c|c|}
\hline Grupo & & $\begin{array}{l}\text { Nunca han comprado un } \\
\text { trabajo académico en } \\
\text { Internet }\end{array}$ & $\begin{array}{l}\text { Han } \\
\text { comprado un } \\
\text { trabajo en } \\
\text { Internet y lo } \\
\text { han } \\
\text { entregado sin } \\
\text { cambios como } \\
\text { si fuera } \\
\text { propio entre } 1 \\
\text { y } 2 \text { veces }\end{array}$ & $\begin{array}{l}\text { Han } \\
\text { comprado un } \\
\text { trabajo en } \\
\text { Internet y lo } \\
\text { han } \\
\text { entregado sin } \\
\text { cambios como } \\
\text { si fuera } \\
\text { propio entre } 3 \\
\text { y } 4 \text { ocasiones }\end{array}$ & $\begin{array}{l}\text { Han } \\
\text { comprado un } \\
\text { trabajo en } \\
\text { Internet y } 10 \\
\text { han } \\
\text { entregado sin } \\
\text { cambios como } \\
\text { si fuera } \\
\text { propio en } 5 \text { o } \\
\text { más de } 5 \\
\text { ocasiones }\end{array}$ \\
\hline $\begin{array}{l}\text { Grupo } \\
\text { Alumnado UIB }\end{array}$ & 1 & $95,3 \%$ & $3,8 \%$ & $0,8 \%$ & $0,1 \%$ \\
\hline $\begin{array}{l}\text { Grupo } \\
\text { Alumnado } \\
\text { registrado } \\
\text { UNIVERSIA }\end{array}$ & en & $96,8 \%$ & $2,7 \%$ & $0,3 \%$ & $0,2 \%$ \\
\hline
\end{tabular}

El $32,3 \%$ del alumnado encuestado de la UIB y el $27,1 \%$ de los alumnos registrados en UNIVERSIA consideran que sus compañeros sí han comprado trabajos académicos a través de Internet, al menos en una ocasión (ver Tabla 4). 
Tabla 4: Compra de trabajos académicos de Internet lopinión de las prácticas del resto de alumnado)

\begin{tabular}{|c|c|c|c|c|}
\hline Grupo & $\begin{array}{l}\text { Creen que sus } \\
\text { compañeros } \\
\text { nunca han } \\
\text { comprado un } \\
\text { trabajo en } \\
\text { Internet y lo } \\
\text { han entregado } \\
\text { sin cambios } \\
\text { como si fuera } \\
\text { propio }\end{array}$ & $\begin{array}{l}\text { Creen que sus } \\
\text { compañeros } \\
\text { esporádicamente } \\
\text { han comprado un } \\
\text { trabajo en Internet } \\
\text { y lo han entregado } \\
\text { sin cambios como } \\
\text { si fuera propio. }\end{array}$ & $\begin{array}{l}\text { Creen que sus } \\
\text { compañeros } \\
\text { frecuentemente } \\
\text { han comparado } \\
\text { un trabajo en } \\
\text { Internet y lo han } \\
\text { entregado sin } \\
\text { cambios como si } \\
\text { fuera propio }\end{array}$ & $\begin{array}{l}\text { Creen que sus } \\
\text { compañeros muy } \\
\text { frecuentemente } \\
\text { han comprado un } \\
\text { trabajo en } \\
\text { Internet y lo han } \\
\text { entregado sin } \\
\text { cambios como si } \\
\text { fuera propio }\end{array}$ \\
\hline $\begin{array}{l}\text { Grupo } 1 \\
\text { Alumnado UIB }\end{array}$ & $67,7 \%$ & $27,3 \%$ & $3,6 \%$ & $1,4 \%$ \\
\hline $\begin{array}{lr}\text { Grupo } & 2 \\
\text { Alumnado } & \\
\text { registrado } & \text { en } \\
\text { UNIVERSIA } & \end{array}$ & $72,9 \%$ & $19,2 \%$ & $5,1 \%$ & $2,8 \%$ \\
\hline
\end{tabular}

\section{3) Conclusiones y discusión}

En las páginas anteriores se ha mostrado cómo el desarrollo de Internet ha provocado profundos cambios en la forma de comprar y vender trabajos académicos para utilizarlos de forma fraudulenta. También se ha puesto de manifiesto cómo en la actualidad nos enfrentamos a una nueva forma de fraude mediante la suplantación de la autoría de los trabajos académicos.

En cuanto a los resultados obtenidos durante el proceso de investigación que se ha presentado es destacable el hecho de que se encuentran en sintonía clara con los datos obtenidos por otras investigaciones similares desarrolladas. Es, asimismo, interesante atender al hecho de que la prevalencia en la comisión de prácticas académicas deshonestas es muy superior cuando el alumnado responde a la consideración de lo que cree que hacen el resto de sus compañeros que cuando la respuesta va orientada a describir sus acciones en primera persona.

Sin duda es éste un problema serio que puede detectarse en muchos países. En un reciente informe de la Inspection générale de l'administion de l'Éducation nationale et de la Recherche (Gauthier, Caffin-Ravier, Descamps B., Mosnier, \& Peretti, 2007) sobre la evaluación de los 
estudiantes en la Universidad francesa, se preguntan los autores si hay que preocuparse por el fraude académico. Su respuesta es contundente: después de analizar cómo se evalúa al alumnado en la Universidad, la comisión constató que nos enfrentamos a un desafío serio y que, además "la fraude tendra à se developer". Como ya hemos señalado, el problema no se circunscribe a un solo país; autores como Hallack y Poisson (2006), que han estudiado el tema en ámbitos geográficos más amplios, pronostican que el rápido crecimiento de la demanda en la acreditación de niveles propios de educación superior y la incapacidad de darle respuesta de una forma satisfactoria puede favorecer la propagación del fraude. La existencia de numerosos portales que hemos descrito en este trabajo, portales que incitan al alumnado, ya sea de secundaria o universitario, a comprar los trabajos académicos es, ya de por sí, una demostración de que nos encontramos frente a un problema serio.

A partir del análisis realizado creemos importante seguir trabajando sobre el tema con el objetivo de poder aportar respuestas a cuestiones como: ¿Seguirá aumentando el número de alumnos que adquieren trabajos ya realizados? ¿Cómo se puede combatir esta plaga? ¿Se producirá en Europa la proliferación de portales de trabajos académicos a la carta como se da en EEUU? ¿Debe legislarse para poner freno a estos portales? ¿Supondrá la creación del Espacio Europeo de Educación Superior una mayor proliferación del fraude de los trabajos a la carta?

Nos resignamos a aceptar, como afirmaba un personaje de Torgny Lindgren (Lindgren, 2007), que "eso que llamamos falsificaciones es la única expresión verdadera de nuestra época". Queremos creer que la mercantilización de la función educativa de la Universidad (Noble, 1997) no acabará con los principios éticos y morales que, precisamente, la Universidad tiene obligación de proteger, inculcar y difundir. En todo caso, para que la mercantilización no arrase estos principios se hace urgente no dejar de estudiar fenómenos como el de las fábricas de ensayos y, sobre todo, introducir la dimensión ética en la formación del alumnado universitario.

\section{Referencias:}

- Campbell, C. R., Swift, C. O., \& Denton, L. (2000): Cheating goes hi-tech: Online term paper mills. Journal of Management Education, 24(6) 726-739.

- Comas, R., \& Sureda, J. (2007): Ciberplagio académico, una aproximación al estado de los conocimiento. Textos de la Cibersociedad, 10. Accedido el 20 Mayo de 2008 Accesible desde http://cibersociedad.net/textos/articulo.php?art=121

- Comas, R., Sureda, J., \& Urbina, S. (2005, 2005): The "copy and paste" generation. plagiarism amongst students. A review of existing literature. The International Journal of Learning, Volumen 12. ISSN: 1477-8386

- Eco, U. (2001): Cómo se hace una tesis (6a ed.). Barcelona: Gadisa. 
- Fain, M., \& Bates, P. (2003): Cheating 101: Paper mills and you. Accedido el 23 de Mayo de 2008. Accesible desde: http://www.coastal.edu/library/presentations/papermil.html

- Gauthier, R. F., Caffin-Ravier, M., Descamps B., Mosnier, M., \& Peretti, H. (2007): L'évaluation des étudiants à l'université: Point aveugle ou point d'appui? (Informe dirigido a la ministra de enseñanza superior e investigación No. 2007-072). Paris: Inspection générale de l'administration de l'éducation nationale et de la recherche. Accedido el 20 de Mayo de 2008. Accesible desde: http://www.education.gouv.fr/cid5592/l-evaluation-des-etudiantsa-I-universite-point-aveugle-ou-point-d-appui.html

- Hallak, J., \& Poisson, M. (2006). Fraude académico, acreditación y garantía de la calidad: Lecciones aprendidas del pasado y retos para el futuro. In Global University Network for Innovation (GUNI) (Ed.), La educación superior en el mundo 2007 (pp. 109-134). Madrid: Ediciones Mundi Prensa.

- Hansen, A. (2003, Combating plagiarism. CQResearcher, 13 (Vol. 32) 773-796.

- HERO. (2007). Essays for sale. Accedido el 20 de Mayo de 2008. Accesible desde: http://hero.ac.uk/uk/inside_he/essays_for_sale.cfm

- Leland, B. (2002). Plagiarism and the web. Accedido el 23 de Mayo de 2008. Accesible desde: http://www.wiu.edu/users/mfbhl/wiu/plagiarism.htm

- Levinson, H. (2005, 14 abril 2005). Essay sales "belittle education". [Versión Electrónica]. $B B C$ News, Accedido el 1 de Junio de 2008. Accesible desde: http://news.bbc.co.uk/2/hi/uk_news/education/4445357.stm.

- Lindgren, T. (2007). En elogio de la verdad [Till sanningens lov] (M. Torres Trans.). Madrid: Nórdica libros.

- Maxymuk, J. (2006): The persistent plague of plagiarism. The Bottom Line: Managing Library Finances, 19 (Vol. 1) 44-47.

- McCabe, D. (2001): Cheating: Why do students do it and how can help them to stop. American Educator, 25 (Vol. 4) 38-43.

- Noble, D. F. (1997): Digital Diploma Mills, Part I. The Automation of Higher Education. Accedido el 15 Mayo de 2008. Accesible desde: http://communication.ucsd.edu/dl/ddm1.html

- Scanlon, P. M., \& Neumann, D. R. (2002): Internet plagiarism among college students. Journal of College Students Develpment, (Mayo/Junio) 375-384. Accedido el 20 de Mayo de $2008 . \quad$ Accesible desde: http://findarticles.com/p/articles/mi_qa3752/is_200205/ai_n9081221 
- Université de Lyon. (2007): Enquête sur les usages d'internet à l'université de lyon. de la documentation au plagiat. Lyon: Université de Lyon. Accedido el 27 Mayo de 2008. Accesible desde: http://www.compilatio.net/enquete.php

- Waugh, E. (2007). Una educación incompleta [A Little Learning: The First Volume of an Autobiography] (M. Martínez-Lage Trans.). Barcelona: Libros del Asteroide.

- Whipple, C. (Producer). (2004). Caught cheating. [Motion Picture] ABC Programa Primetime. Accedido el 5 de Junio de 2008. Accesible desde: http://www.youtube.com/watch?v=hoH4yqVVp8c

\section{Para citar este artículo:}

COMAS, Rubén; SUREDA, Jaume (2008) «El intercambio y compra-venta de trabajos académicos a través de internet» [artículo en línea]. EDUTEC, Revista Electrónica de Tecnología Educativa. Núm. 26/Julio 2008. [Fecha de consulta: $\mathrm{dd} / \mathrm{mm} / \mathrm{aa}$ ].

http://edutec.rediris.es/revelec2/revelec26/

ISSN 1135-9250. 